\title{
Expression Profile and Prognostic Value of $C X C R$ Family Members in Head and Neck Squamous Cell Carcinoma
}

\section{Yiming Shen}

Ningbo University

Chongchang Zhou

Ningbo Medical Center Lihuili Hospital

\section{Yujie Cao}

Ningbo Medical Center Lihuili Hospital

Qun Li

Ningbo Medical Center Lihuili Hospital

Hongxia Deng

Ningbo Medical Center Lihuili Hospital

Shanshan Gu

Ningbo Medical Center Lihuili Hospital

\section{Yidong Wu}

Ningbo Medical Center Lihuili Hospital

Zhisen Shen ( $\nabla$ szs7216@163.com )

Ningbo Medical Center Lihuili Hospital

\section{Research Article}

Keywords: Head and neck squamous cell carcinoma, CXC chemokine receptor gene family, the cancer genome atlas, prognosis, survival

Posted Date: November 17th, 2021

DOI: https://doi.org/10.21203/rs.3.rs-1078583/v1

License: (c) (i) This work is licensed under a Creative Commons Attribution 4.0 International License. Read Full License 


\section{Abstract}

Background: CXC chemokine receptor gene family consists of seven well-established members which are broadly involved in biological functions of various cancers. Currently, limited studies have shed light on the expression profile of CXCR family members (CXCRs), as well as their prognostic value, in head and neck squamous cells carcinoma (HNSCC).

Methods: The data for this study were retrieved from the Cancer Genome Atlas database and other publicly available databases, including gene expression, methylation profiles, clinical information, immunological features and prognoses. The expression pattern and prognostic values of CXCRs were identified, and the potential mechanism underlying CXCRs function in HNSCC was investigated by gene set enrichment analysis (GSEA).

Results: CXCRs were differentially expressed in HNSCC. As shown by Kaplan Meier analysis, high CXCR36 expression was significantly associated with better prognostic outcomes of HNSCC patients, including overall survival and progression-free survival. According to the results of univariate and multivariate Cox proportional risk regression analysis, it was demonstrated that upregulation of CXCR3-6 was an independent factor for better prognosis, while the two other clinical features, age and stage, were factors for worse prognosis. A significant positive correlation between CXCR3-6 and tumor-infiltrated immune cells was revealed by results from Tumor Immune Estimation Resource and CIBERSORT analysis database. The main involvement of CXCRs in immune and inflammatory responses was further confirmed by GSEA.

Conclusions: Overall, this study provided a rationale for targeting CXCRs as a promising therapeutic strategy of HNSCC.

\section{Background}

Head and neck squamous carcinoma (HNSCC) is the most common pathological subtype of head and neck cancer which is a highly aggressive malignancy (1). The annual number of people diagnosed with head and neck cancer worldwide has reached nearly 500,000, and the mortality rate of the patients diagnosed with HNSCC has increased over the last few years (2). Although prominent progress has been made in the treatment of HNSCC, the 5-year survival rate of HNSCC patients remains around $50 \%$ (3). Therefore, further understanding of the complex cancer biology underlying this disease and developing more reliable predictive biomarkers for HNSCC prognosis is critical for facilitating the therapeutic efficacy of individualized treatment, which may ultimately help to enhance patients' survival.

With more than 50 members, vertebrate $\mathrm{G}$ protein-coupled chemokine receptors are comprised of four different subfamilies, including CC chemokine receptors, XC chemokine receptors, CXC chemokine receptors and $\mathrm{CX} 3 \mathrm{C}$ chemokine receptors. CXC chemokine receptors are a large subfamily of chemokine receptors, which consists of seven members known as $C X C R 1$ to $C X C R 7$ (4). CXCR family members (CXCRs) are well-known to participate in both anti-tumor immune response and tumorigenesis (5). CXC 
chemokine receptors 1 and 2 (CXCR 1 and $C X C R 2)$, members of the receptors of ELR ${ }^{+}$chemokines, are generally expressed simultaneously on neutrophils, fibroblasts and vascular endothelial cells, and have a relation to the activation of multiple downstream signaling pathways. A high affinity exhibited by both CXCR1 and CXCR2 has been observed toward the CXC motif chemokine ligand (CXCL) 8, a common ligand secreted by cancer cells and inflammatory cells. Binding of the ligand to these receptors promotes tumor invasion, angiogenesis, and metastasis $(6,7)$. CXCR3-6 are the receptors of the ELR ${ }^{-}$chemokines (8). CXCR3 can bind to CXCL9, CXCL10, and CXCL11 respectively, and its expression can be detected on various subtypes of natural killer (NK) cells and T cells (9-12). CXCR3 exhibits diverse biological functions upon extracellular stimuli which activate intracellular signal transduction pathways that involved in survival, cell behavior, and cell identity. CXCR4, an important regulator of the homing and mobilization of hematopoietic cell, is widely expressed on different cell types like megakaryocytes, embryonic stem cells, vascular endothelial cells, and cancer cells $(13,14)$. The interaction of CXCR4 with its ligand, $C X C L 12$, has been shown to facilitate the migration of $\mathrm{T}$ - and $\mathrm{B}$-lymphocyte. The $C X C R 5$ is a receptor for CXCL 13 which is a homeostatic chemokine. Upon contact with CXCL13, CXCR5 is capable of regulating cell migration and $\mathrm{GC}$ reactions (15). The overexpression of $C X C R 5$ and its cognate ligand CXCL13 has been implicated in many different types of cancer (16). Expressed on CD8 ${ }^{+} \mathrm{T}, \mathrm{NK}$, and CD4 ${ }^{+}$ T cells, CXCR6 could promote the recruitment of tumor-infiltrating lymphocytes in combination with CXCL 16 expressed by tumor cells, thus contributing to a better prognosis for cancer patients (17). CXCR7, previously known as $A C K R 3$. Although it is also a seven-transmembrane receptor, $C X C R 7$ cannot mediate the classical Gi signaling pathway like CXCR3 and many other chemokine receptors (18). CXCR7 has previously been regarded as a "decoy" and an atypical chemokine receptor for CXCL 12. Moreover, CXCR7 is a promising therapeutic target with its involvement in tumorigenesis and autoimmune disorders. However, the mechanism by which CXCRs are activated or deactivated in the development and progression of HNSCC remains unclear until now.

Here, we comprehensively analyzed the expression of the whole CXC chemokine receptor gene family and the potential correlation with prognostic value and clinical significance in HNSCC via different databases, including Oncomine and the Cancer Genome Atlas (TCGA), which provide detailed information about cancers. In addition, Tumor Immune Estimation Resource (TIMER) was employed to investigate the association between CXCRs and immune infiltration degree. To determine HNSCC-related risk factors, univariate/multivariate Cox regression models were utilized, and GSEA about CXCRs-related signaling pathways was performed.

\section{Methods}

\subsection{Data resources}

HNSCC transcriptomic sequencing data of 502 HNSCC and 44 normal samples were acquired from TCGA data (https://portal.gdc.cancer.gov/) (19). The clinical features from the extracted clinical information of patients were included in the present study, including gender, age, stage, and tumor grade. Subsequently, 
the high-throughput sequencing (HTSeq) Fragments Per Kilobase of transcript per Million mapped reads (FPKM) data of CXCRs were download by utilizing Genomic Data Commons (GDC) Data Transfer Tool on the TCGA database.

\subsection{Expression data of CXCRs in HNSCC}

Gene expression of CXCRs was analyzed across multiple datasets by comparing tumor tissues to the healthy tissues, using Oncomine (http://www. oncomine.org) (20). And the thresholds were set as follows: gene rank was set in the top $10 \%$; data type was limited to mRNA; $p$-value was set to 0.01 and folding change (FC) was defined as 1.5. Based on the HTSeq FPKM data, Perl 5.26 software was adopted to extract the mRNA expression levels of CXCRs in HNSCC. The limma package in R software was employed to analyze how CXCRs were expressed differentially in HNSCC tissues and normal tissues. The pheatmap and ggpubrpackages were utilized for the visualization of a heatmap and box plots to display analysis results.

\subsection{Relationship between methylation and mRNA expression of CXCRs in HNSCC}

Data on the methylation levels of $\mathrm{cg}$ sites, which were within the gene promoter regions of differentially expressed CXCRs in HNSCC tissues, was download from Illumina Human Methylation 450K through GDC Data Transfer Tool on the TCGA, and then was annotated with the annotation file from the official Illumina website (https://support.illumina.com/downloads/ infinium_humanmethylation450_product_files.html). After the methylation levels of $\mathrm{cg}$ sites were identified in CXCRs gene promoter regions, the corrplot package was utilized to understand how the methylation level and CXCR expression in HNSCC correlated.

\subsection{Prognostic value evaluation of CXCRs in HNSCC}

The Kaplan-Meier method was adopted for the analysis of the survival rate. R package survival analysis was implemented combined with gene expression profiles and survival information of HNSCC involving a total of 499 HNSCC patients, to evaluate how the mRNA expression of CXCR1-7 correlated with the prognoses of HNSCC patients. Then the progression-free survival (PFS) was assessed along with overall survival (OS) time in search of the best optimal cutoff value. Moreover, for the estimation of the independent prognostic factors, we utilized univariate and multivariate Cox proportional hazards models. And the forest plots were created to present the analysis results by using the ggplot package.

\subsection{Pearson correlation and protein-protein interaction (PPI) network of CXCRs}


To determine the correlation among $C X C R s$, a Pearson correlation analysis was carried out using the corrplot package of $\mathrm{R}$ software genes on the basis of the gene expression data obtained from the TCGA database. In order to retrieve interacting genes or proteins, we established the PPI networks of CXCRs by the Search Tool (STRING, http:// www.string-db.org/).

\subsection{Gene set enrichment analysis (GSEA)}

To further investigate how CXCRs participated in HNSCC carcinogenesis, GSEA was carried out (version 4.0.1; http://software. broadinstitute.org/gsea/index.jsp) to identify the CXCRs- associated signaling pathways in the TCGA HNSCC tissues $(21,22)$. The identification of significant enrichment pathways employed a false discovery rate (FDR) of $<0.05$ and a random combination number of 1,000 for each mutation, with the reference to the annotated gene set file from the Msig database (c2.cp.kegg.v7.0.symbols.gmt).

\subsection{Corrections between tumor immune infiltrating cells (TIICs) and CXCRs}

During cancer progression, tumor cells interact with TIICs through a variety of genes and pathways. To explore the correlation of TIICs with CXCRs, the TIMER platform was adopted for analysis (https://cistrome.shinyapps.io/timer/), which is an online database incorporating expression profiles of over 10,000 samples across 23 cancer types from TCGA $(23,24)$. According to TIMER, macrophages, CD4+ T-cells, neutrophils, dendritic cells, B-cells, and CD8+ T-cells are the main components of the TIICs. The purity-corrected partial Spearman's correlation (partial-cor) provided by TIMER was presented together with $\mathrm{p}$-value in scatterplots in the current study. On the basis of the median value of gene expression, we divided the samples into high expression group and low expression group. The vioplot package of $\mathrm{R}$ software was utilized to visualize the analysis results. In a mixed cell population, the specific proportions that 22 tumor-infiltrating lymphocyte subsets accounted for respectively were estimated by the analytical tool CIBERSORT with gene expression data (https://cibersort.stanford.edu/).

\section{Results}

\subsection{Differential expression of CXCRs in HNSCC Patients}

It has been implicated that the aberrant expression of CXCRs exists in various cancer types like leukemia, lymphomas and lung cancer. To assess the expression pattern of CXCRs in HNSCC, the gene expression data from Oncomine was analyzed. Oncomine analysis results shown in Figure 1A demonstrated that mRNA expression levels of CXCR4 and CXCR7 were upregulated in all subtypes of Head and Neck cancer, while $C X C R 2$ and $C X C R 5$ were downregulated (fold change $>1.5$, $\mathrm{p}$-value $<0.05$ ). And the mRNA expression level of $C X C R 4$ was notably upregulated in HNSCC tissue when compared with the healthy tissue (Table 
1). Consistently, a previous study found that CXCR4 was shown to be overexpressed in HNSCC tissues in comparison to the healthy tissues, with a Fold Change (FC) of 3.447 ( $p$-value $=2.75 E-13)$ (25). Also, in another study, the mRNA level of CXCR4 was found to have a 2.150-fold increase in HNSCC tissue (pvalue $=4.68 \mathrm{E}-6)(26)$. Moreover, a significantly high level of $C X C R 7$ mRNA expression was previously found in HNSCC tissues in other studies. For example, the FC of the CXCR7 expression in HNSCC tissue was 2.185 (p-value $=3.24 \mathrm{E}-7$ ) and 6.647 ( $p$-value $=0.002$ ), respectively in the study by Matthew et al and Gokce et al. $(25,27)$. The significant difference between the transcription level of $C X C R 7$ in HNSCC and that in healthy head and neck tissues was revealed in another study (28). In addition, 1.550-fold increase $(p$-value $=1.28 \mathrm{E}-10)$ and 2.378 -fold increase $(p$-value $=1.49 \mathrm{E}-4)$ in the $C X C R 6$ expression in HNSCC tissues were found in two other studies $(26,29)$. For the further understanding of the expression profile of CXCRs in HNSCC, based on 502 HNSCC samples and 44 healthy control samples from the TCGA database, Perl software and the limma package were utilized to obtain mRNA expression data of CXCR members (CXCR1-7) and analyze the differentially expressed CXCRs. The differential gene expression of CXCRs in the HNSCC samples, as well as normal tissues, was displayed in the heatmap generated by the pheatmap package (Figure 1B). Furthermore, the aberrant expression levels of all CXCR members in HNSCC compared to the healthy tissues were revealed by the analysis results, especially the significant downregulation of $C X C R 2$ in HNSCC tissues. While other the CXCRs, namely CXCR1 and CXCR3 to $C X C R 7$, were significantly upregulated in HNSCC tissues (Figure 1C).

\subsection{Methylation of the promoter regions of CXCR genes in HNSCC}

In the progression of cancer, one of the most typical mechanisms having a critical impact on gene expression is methylation of gene promoter regions. As Pearson's correlation analysis indicated, among the identified seven $C X C R$ members in HNSCC, five of the $C X C R$ members, including $C X C R 2$, and CXCR4 to CXCR7 were negatively correlated with methylation level (Figure 2B-F). Moreover, of the 4 evaluated cg sites in the CXCR1 promoter region, only 2 were negatively associated with the expression of CXCR1 in HNSCC (Figure 2A). Together, the expression of CXCR members was inversely correlated with their methylation level in HNSCC according to the existing data.

\subsection{Prognostic value of CXCRs in HNSCC}

To determine the correlation of $C X C R$ family genes expression with the survival of HNSCC patients, how mRNA expression of CXCRs correlated with overall survivals on log-rank test was examined using Cutoff finder. The overall survival and the progression-free survival in patients with $C X C R 1-7$ expression were plotted by utilizing Kaplan-Meier curves (Figure 3). A significant association was observed between high mRNA expression of $C X C R 3$ to $C X C R 6$ and favorable overall survival (Figure 3C-F). Furthermore, there was a trend toward improved relapse-free survival with the high expression level of $C X C R 3$ to $C X C R 6$, with statistical significance (log-rank test $\mathrm{P}<0.01$, Figure $3 \mathrm{H}-\mathrm{L}$ ). Subsequently, we aimed to determine the 
prognostic influences of $C X C R$ members in HNSCC. To evaluate the predictive ability of differentially expressed $C X C R s$ and their correlation with clinical characteristics, univariate Cox proportional hazards regression analysis was used. The association between the expression of four $C X C R$ members (CXCR3 to CXCR6) and the favorable outcome of HNSCC patients was found, so as the association of two clinical features (age and stage) with poor outcome (hazard ratio [HR] for CXCR3: 0.649; HR for CXCR4: 0.529; HR for CXCR5: 0.623; HR for CXCR6: 0.650; HR for age: 1.024; and HR for stage: 1.448) (Table 2). In the evaluation of the independent prognostic values of CXCR3 to CXCR6 along with the controlling of prognostic effects of these clinical features, the independent prognostic biomarkers of HNSCC outcome were reported by multivariate Cox proportional hazards regression analysis, i.e., the expression of $C X C R 3$ to CXCR6 and two clinical parameters (age and stage) (Figure 4).

\subsection{Potential molecular mechanism underlying the roles of prognostic CXCRs in HNSCC}

On the basis of the gene expression data from TCGA, a PPI network was established by a Pearson correlation analysis combined with STRING database, aiming to determine the related protein interactions and possible correlation among $C X C R$ family genes. A strong correlation was found between the $C X C R s$ and interleukin-8 receptor activity (Figure 5A), as well as the CXCR1 gene and CXCR2 (Figure 5B). Besides, regarding the underlying biological mechanism of how the carcinogenesis of HNSCC is mediated by differential expression of $C X C R 3$ to $C X C R 6$, a GSEA was carried out on the differentially expressed $C X C R S$ with statistical prognostic value. 10 common cell signaling pathways closely related to high expression of $C X C R 3$ to $C X C R 6$ were proposed by the GSEA (Figure $6 \mathrm{~A}$ ), including "hematopoietic cell lineage", "B cell receptor signaling pathway", "JAK-STAT signaling pathway", "cell adhesion molecules cams", "leukocyte trans-endothelial migration", "chemokine signaling pathway", "natural killer cell-mediated cytotoxicity", "cytokine-cytokine receptor interaction", "leishmania infection", and "T cell receptor signaling pathway" (Figure 6B-E).

\subsection{Associations between TIICs and CXCRs in HNSCC}

Given the growing evidence on the association between cancer prognosis and immunological features, the potential relevance of $C X C R$ members in TIICs in HNSCC was further explored. For the evaluation of the association between immune cells surrounding tumor cells and certain gene products, we made use of the public resource provided by the TIMER database. The scatterplots were shown here presenting the expression of $C X C R$ members against tumor purity. It was reported that in stromal-immune cells surrounding tumor cells, the high expression of $C X C R s$ was anticipated to be negatively correlated with tumor purity, but positively correlated in tumor cells. Consistent with the results mentioned above, CXCR1 to CXCR6 were highly expressed in HNSCC tissues, which showed a negative correlation with tumor purity (Figure 7) and suggested that there was a significant correlation between TIICs and CXCR members, CXCR3-6 in particular. The relationship between CXCRs gene expression and the abundance of 22 
immune cell types in HNSCC was revealed by CIBERSORT results (Figure 8). High CXCR3 expression was significantly related with more regulatory $T$ cells (Tregs), M0 macrophages, naive $B$ cells, resting mast cells, follicular helper T cells, activated dendritic cells, activated mast cells, memory B cells, activated memory CD4+ T cells, M1 macrophages, CD8+ T cells, and less naive CD4+ T cells, Eosinophils infiltration. High CXCR4 expression was associated with gamma delta T cells, more naive B cells, activated NK cells, Tregs, activated memory CD4+ T cells, M2 macrophages, follicular helper T cells, activated dendritic cells, and less plasma cells, CD8+ T cells, resting NK cells, M0 macrophages, activated mast cells, Eosinophils infiltration. High CXCR5 expression was positively related with follicular helper $T$ cells, naive B cells, Tregs, plasma cells, gamma delta T cells, CD8+ T cells, resting NK cells, resting mast cells, memory B cell, and negatively related with M0 macrophages, activated mast cells, Eosinophils, activated memory CD4+ T cells, activated NK cells. Moreover, it was also demonstrated that high CXCR6 expression was positively related with activated memory CD4+ T cells, naive B cells, Tregs, CD8+ T cells, resting NK cells, follicular helper T cells, and negatively related with activated mast cells, M1 macrophages, resting mast cells, Eosinophils, activated dendritic cells, M0 macrophages. Taking these findings together, $C X C R$ family members may play a crucial role in the regulation of the immune microenvironment in HNSCC, with the potential to make promising targets for the treatment of HNSCC patients.

\section{Discussion}

CXC chemokine receptors represent a large subfamily of the G protein-coupled receptors superfamily. Chemokine receptors, as the name suggests, have the primary function of orchestrating cell trafficking, in particular, the mobilization of immune cells to the sites of inflammation. Although the role of the $C X C R$ gene family in the onset and progression of many human cancers has been highlighted by accumulating evidence, including breast cancer, bladder cancer, acute lymphoblastic leukemia (ALL), and acute myeloid leukemia (AML) (30-32), the exact prognostic values of CXCRs in HNSCC remain to be further explored. With increasing published or publicly available genomic data and a variety of online platforms, it is possible to investigate the expression profiles and clinical practice value of families of genes in human cancers. In the current study, the distinct expression profile, methylation and prognostic values of CXCRs were clarified, as well as their involvement in various biological processes in HNSCC. We carried out the evaluation of the CXCRs expression levels in different malignancies and found the high expression of $C X C R 4$ and $C X C R 7$ in HNSCC samples from ONCOMINE. Moreover, TCGA data analysis implicated the high expression of $C X C R 1$ to $C X C R 7$ in HNSCC tissues compared to the healthy tissues. Although $C X C R s$ have been confirmed to be highly expressed in various tumor tissues by previous research, this study is the first to present the expression of $C X C R 1$ to $C X C R 7$ in HNSCC. The methylation levels in promoter areas were further analyzed to explore the underlying mechanism of abnormal CXCRs expression in HNSCC. Genes are involved in apoptosis, cell proliferation, and cell cycle through gene silencing and reactivation, which can be caused by demethylation and methylation of $\mathrm{cg}$ sites in promoter regions. The regional context along with neighboring sites is concerned with methylation changes at individual cg site. According to Pearson's correlation analysis, among the six 
differentially expressed $C X C R$ members (CXCR1, CXCR2 to CXCR7), the expression level of CXCR4 is particularly affected by its methylation level, suggesting the possible crucial role of abnormal methylation in the aberrant expression of these genes. Yet, the abnormal expression of the $C X C R$ gene family may also result from other epigenetic or genetic alterations like gene mutations and copy number changes. No significant correlation of mRNA expression levels of CXCR1 and CXCR2 with OS and RFS was found in this study, while high expression of $C X C R 3 / 4 / 5 / 6$ turned out to be related to favorable survival outcomes in patients with HNSCC. Univariate and multivariate Cox analyses were carried out combined with parameters of age, stage, and the 7 identified hub genes, in the hope of establishing a relatively accurate prognostic model for patients with HNSCC. Our analysis data suggested the predictive potential of $C X C R$ as four $C X C R$ members (CXCR3, CXCR4, CXCR5, and CXCR6) were significantly related to better clinical outcomes in HNSCC, as reported in acute myeloid leukemia (33). The underlying molecular mechanisms of abnormal expression of $C X C R s$ regulating HNSCC carcinogenesis were further studied. The significant correlation among CXCRs and their vital role in the interaction with CXC motif chemokine 8/9/10/12/13 were clarified by Pearson's correlation analysis and the PPI network. CXCRs expression was also concerned with some signaling pathways involved in cancer progression, as indicated by the GSEA, thus leading to the potential mechanism of CXCR carcinogenicity. The significant correlation between $C X C R 3$ to $C X C R 6$ and cytokine-cytokine receptor interaction as well as was cell adhesion molecules cams also worth noting, which indicated an interaction with CXC motif chemokine. The association between $C X C R$ family genes and TIICs was observed by the TIMER and CIBERSORT analyses, offering favorable proof for the connection between CXCRs expression and the immune microenvironment in HNSCC. Taken together, our data supported the regulatory function of $C X C R s$ family members in the HNSCC immune microenvironment, which deserves further research.

It is already known that $C X C R 3$ is primarily expressed on vascular cells, NK cells, tumor cells, and activated T lymphocytes, and binds to $C X C L 9$ to $\operatorname{CXCL} 11(10,11)$. CXCR3 has three different isoforms in humans, CXCR3A, CXCR3B, and $C X C R 3 A / t$, and its dual role in immune response and cancer has been reported. For example, in a previous study, CXCR3 was found to be the key mediator of tumorigenesis, and the main cause of poor response and early recurrence in multiple cancers in multiple cancers (34). Conversely, another study reported that CXCR3-CXCL9/10/11 signaling pathway contributed to the chemotactic movement of immune cells activated by CXCR3 to the tumor site, thereby promoting the antitumor immune response (35). The data presented here verified that CXCR3 expression was positively associated with immune cell infiltration, suggesting the overexpression of $C X C R 3$ could probably increase the infiltration of immune cells of all sorts into the tumor microenvironment.

The crystal structures of $C X C R 4$ reveals the way chemokine receptors are regulated by various modulators binding to different or sometimes overlapping binding pockets. CXCR4 has been implicated in the onset of a great number of tumors, due to the reason that this receptor is considered to be crucial in immune cells chemotaxis, tumor cell proliferation, invasion, metastasis and angiogenesis. Widely expressed in physiological conditions, CXCR4 and its ligand CXCL 12 are essential regulators of hematopoiesis, cardiogenesis, and neurogenesis. 
As a specific receptor of CXCL13, CXCR5 mediates cancer functions regulated by CXCL13(36). According to Singh et al., the expression of CXCR5 was increased significantly only in squamous cell carcinoma (SCC) with lung node metastasis, but not in other subtypes of lung cancer (37). Moreover, Ma et al. reported the contribution of CD4+CXCR5+ T cells to anti-tumor immunity and its relation to better outcomes in nonsmall-cell lung cancer (38).

CXCR6 expression was discerned in different hepatoma cell lines with various metastatic properties. Also, CXCR6 contributes to the microenvironment by inducing inflammatory cytokines, leading to the invasion and metastasis of hepatoma cells (39). The high expression of CXCR6 in HNSCC was revealed by TCGA database analysis in this study, especially in HNSCC patients at the advanced stage or the elder. Furthermore, high expression of CXCR6 was proven to be connected to improved OS in HNSCC, and meanwhile, $5 \mathrm{CpGs}$ of CXCR6 make a difference to favorable survival. CXCR6-CXCL 16 axis also recruits immune cells to cancer sites just like the way of other $C X C R s$, thereby affecting the progression of cancer. However, the cell-specific functions of CXCR6 remain unclear to a large extent (40).

Overall, this study performed a comprehensive and thorough analysis of the expression and prognostic values of the CXCRs in HNSCC using publicly available TCGA databases. This study found that some of the $C X C R$ family members could serve as clinical biomarkers of HNSCC. Our findings highlighted the vital function of $C X C R 3$ to $C X C R 6$ in HNSCC progression, as well as their clinical significance in HNSCC. Furthermore, we provided an insight into the role of $C X C R 3$ to $C X C R 6$ in mediating different signaling pathways in HNSCC. Our study also suggested that CXCR3-6 mediated inhibition of HNSCC progression may correlate with $\mathrm{T}$ cell receptor signaling pathway, leukocyte trans-endothelial migration, and natural killer cell cytotoxicity. Nevertheless, the specific role of CXCR3 to CXCR6 in HNSCC requires future exploration.

\section{Conclusions}

In general, despite the promising results achieved, there are still some limitations in the present study. We performed all analyses and obtained the results on the sole basis of data from the TCGA, without verifying the expression levels of CXCRs protein and mRNA in HNSCC as well. Nevertheless, as suggested by our findings, targeting $C X C R$ family members could be a promising therapeutic approach for treating patients with HNSCC.

\section{Abbreviations}

HNSCC: Head and neck squamous carcinoma

CXCRs: CXC chemokine receptor gene family members

GSEA: Gene set enrichment analysis

CXCL: CXC motif chemokine ligand 
NK cells: natural killer cells

TCGA: the Cancer Genome Atlas

HTSeq: high-throughput sequencing

FPKM: Fragments Per Kilobase of transcript per Million mapped reads

GDC: Genomic Data Commons

PFS: progression-free survival

OS: overall survival

PPI: Pearson correlation and protein-protein interaction

FDR: false discovery rate

TIICs: Corrections between tumor immune infiltrating cells

FC: Fold Change

ALL: acute lymphoblastic leukemia

AML: acute myeloid leukemia

Tregs: regulatory $T$ cells

\section{Declarations}

Ethics approval and consent to participate (Not applicable)

Consent for publication (Not applicable)

\section{Competing interests}

The authors declare no conflicts of interest among them.

\section{Availability of data and materials}

The gene expression data and clinical features used for bioinformatics analyses in this study are publicly available on the Cancer Genome Atlas (TCGA) program website (https://portal.gdc.cancer.gov). The authors are solely responsible for interpreting and reporting these data.

\section{Funding}


Ningbo Health Branding Subject Fund(No.PPXK2018-02);Zhejiang Provincial Natural Science Foundation of China(LY19H160014;LQ21H130001;);Ningbo "Technology Innovation 2025" Major Special

Project(2020Z097;2018B10015);Medical and Health Research Project of Zhejiang

Province(2019ZD018;2021KY307);

\section{Author Contributions}

YMS, CCZ and ZSS conceived and designed the study. YMS, CCZ, YJC, QL performed the analyses. HXD, SSG and YDW elaborated all figures and tables. YMS, CCZ and ZSS wrote the main manuscript. The final version of this paper was approved by all authors.

\section{Acknowledgments}

We thank Medina Abdulla for editing the manuscript.

\section{References}

1. F Bray, Ferlay J, Soerjomataram I, Siegel R L, Torre L A Jemal A. Global cancer statistics 2018: GLOBOCAN estimates of incidence and mortality worldwide for 36 cancers in 185 countries. $C A$ Cancer J Clin. (2018) 68:394-424. doi:10.3322/caac.21492

2. F Peter, Wittekindt C, Finkensieper M, Kiehntopf M Guntinas-Lichius O. Prognostic impact of pretherapeutic laboratory values in head and neck cancer patients. J Cancer Res Clin Oncol. (2013) 139:171-8. doi:10.1007/s00432-012-1320-1

3. S S Lin, Massa S T Varvares M A. Improved overall survival and mortality in head and neck cancer with adjuvant concurrent chemoradiotherapy in national databases. Head Neck. (2016) 38:208-15. doi:10.1002/hed.23869

4. M Sun, Sun L, Sun D, Zhang C Li M. Targeted delivery of immuno-RNase may improve cancer therapy. Cancer Cell International. (2018) 18:58. doi:10.1186/s12935-018-0546-7

5. A Ben-Baruch. The multifaceted roles of chemokines in malignancy. Cancer Metastasis Rev. (2006) 25:357-71. doi:10.1007/s10555-006-9003-5

6. H Ha, Debnath B Neamati N. Role of the CXCL8-CXCR1/2 Axis in Cancer and Inflammatory Diseases. Theranostics. (2017) 7:1543-1588. doi:10.7150/thno.15625

7. W Huang, Chen Z, Zhang L, Tian D, Wang D, Fan D,et al. Interleukin-8 Induces Expression of FOXC1 to Promote Transactivation of CXCR1 and CCL2 in Hepatocellular Carcinoma Cell Lines and Formation of Metastases in Mice. Gastroenterology. (2015) 149:1053-67.e14. doi:10.1053/j.gastro.2015.05.058

8. Q Zhu, Han X, Peng J, Qin H Wang Y. The role of CXC chemokines and their receptors in the progression and treatment of tumors. J Mol Histol. (2012) 43:699-713. doi:10.1007/s10735-0129435-x

9. Y M El-Sherbiny, Kelly R J, Hill A, Doody G M, Hillmen P Cook G P. Altered natural killer cell subset homeostasis and defective chemotactic responses in paroxysmal nocturnal hemoglobinuria. Blood. 
(2013) 122:1887-90. doi:10.1182/blood-2013-06-507574

10. M Lima, Leander M, Santos M, Santos A H, Lau C, Queirós M L,et al. Chemokine Receptor Expression on Normal Blood CD56(+) NK-Cells Elucidates Cell Partners That Comigrate during the Innate and Adaptive Immune Responses and Identifies a Transitional NK-Cell Population. J Immunol Res. (2015) 2015:839684. doi:10.1155/2015/839684

11. F Sallusto, Lenig D, Mackay C R Lanzavecchia A. Flexible programs of chemokine receptor expression on human polarized T helper 1 and 2 lymphocytes. J Exp Med. (1998) 187:875-83. doi:10.1084/jem.187.6.875

12. M T Chow, Ozga A J, Servis R L, Frederick D T, Lo J A, Fisher D E,et al. Intratumoral Activity of the CXCR3 Chemokine System Is Required for the Efficacy of Anti-PD-1 Therapy. Immunity. (2019) 50:1498-1512.e5. doi:10.1016/j.immuni.2019.04.010

13. S Chatterjee, Behnam Azad B Nimmagadda S. The intricate role of CXCR4 in cancer. Adv Cancer Res. (2014) 124:31-82. doi:10.1016/b978-0-12-411638-2.00002-1

14. F Gagliardi, Narayanan A, Reni M, Franzin A, Mazza E, Boari N,et al. The role of CXCR4 in highly malignant human gliomas biology: current knowledge and future directions. Glia. (2014) 62:1015-23. doi:10.1002/glia.22669

15. J F Liu, Lee C W, Lin C Y, Chao C C, Chang T M, Han C K,et al. CXCL13/CXCR5 Interaction Facilitates VCAM-1-Dependent Migration in Human Osteosarcoma. Int J Mol Sci. (2020)

21:doi:10.3390/ijms21176095

16. B Han, Shaolong E, Luan L, Li N Liu X. CircHIPK3 Promotes Clear Cell Renal Cell Carcinoma (ccRCC) Cells Proliferation and Metastasis via Altering of miR-508-3p/CXCL13 Signal. Onco Targets Ther. (2020) 13:6051-6062. doi:10.2147/ott.S251436

17. L Deng, Chen N, Li Y, Zheng H Lei Q. CXCR6/CXCL16 functions as a regulator in metastasis and progression of cancer. Biochimica et Biophysica Acta (BBA) - Reviews on Cancer. (2010) 1806:42-49. doi:10.1016/j.bbcan.2010.01.004

18. I Adlere, Caspar B, Arimont M, Dekkers S, Visser K, Stuijt J,et al. Modulators of CXCR4 and CXCR7/ACKR3 Function. Mol Pharmacol. (2019) 96:737-752. doi:10.1124/mol.119.117663

19. K Tomczak, Czerwińska P Wiznerowicz M. The Cancer Genome Atlas (TCGA): an immeasurable source of knowledge. Contemp Oncol (Pozn). (2015) 19:A68-77. doi:10.5114/wo.2014.47136

20. D R Rhodes, Yu J, Shanker K, Deshpande N, Varambally R, Ghosh D,et al. ONCOMINE: a cancer microarray database and integrated data-mining platform. Neoplasia. (2004) 6:1-6. doi:10.1016/s1476-5586(04)80047-2

21. A Subramanian, Tamayo P, Mootha V K, Mukherjee S, Ebert B L, Gillette M A,et al. Gene set enrichment analysis: a knowledge-based approach for interpreting genome-wide expression profiles. Proc Natl Acad Sci U S A. (2005) 102:15545-50. doi:10.1073/pnas.0506580102

22. V K Mootha, Lindgren C M, Eriksson K F, Subramanian A, Sihag S, Lehar J,et al. PGC-1alpharesponsive genes involved in oxidative phosphorylation are coordinately downregulated in human diabetes. Nat Genet. (2003) 34:267-73. doi:10.1038/ng1180 
23. T Li, Fan J, Wang B, Traugh N, Chen Q, Liu J S,et al. TIMER: A Web Server for Comprehensive Analysis of Tumor-Infiltrating Immune Cells. Cancer Res. (2017) 77:e108-e110. doi:10.1158/00085472.Can-17-0307

24. T Li, Fu J, Zeng Z, Cohen D, Li J, Chen Q,et al. TIMER2.0 for analysis of tumor-infiltrating immune cells. Nucleic acids research. (2020) 48:W509-W514. doi:10.1093/nar/gkaa407

25. M A Ginos, Page G P, Michalowicz B S, Patel K J, Volker S E, Pambuccian S E,et al. Identification of a gene expression signature associated with recurrent disease in squamous cell carcinoma of the head and neck. Cancer Res. (2004) 64:55-63. doi:10.1158/0008-5472.can-03-2144

26. C H Peng, Liao C T, Peng S C, Chen Y J, Cheng A J, Juang J L,et al. A novel molecular signature identified by systems genetics approach predicts prognosis in oral squamous cell carcinoma. PLOS One. (2011) 6:e23452. doi:10.1371/journal.pone.0023452

27. G A Toruner, Ulger C, Alkan M, Galante A T, Rinaggio J, Wilk R,et al. Association between gene expression profile and tumor invasion in oral squamous cell carcinoma. Cancer Genet Cytogenet. (2004) 154:27-35. doi:10.1016/j.cancergencyto.2004.01.026

28. H Ye, Yu T, Temam S, Ziober B L, Wang J, Schwartz J L,et al. Transcriptomic dissection of tongue squamous cell carcinoma. BMC Genomics. (2008) 9:69. doi:10.1186/1471-2164-9-69

29. C L Estilo, O-charoenrat P, Talbot S, Socci N D, Carlson D L, Ghossein R,et al. Oral tongue cancer gene expression profiling: Identification of novel potential prognosticators by oligonucleotide microarray analysis. BMC Cancer. (2009) 9:11. doi:10.1186/1471-2407-9-11

30. I Del Molino Del Barrio, Wilkins G C, Meeson A, Ali S Kirby J A. Breast Cancer: An Examination of the Potential of ACKR3 to Modify the Response of CXCR4 to CXCL12. Int J Mol Sci. (2018) 19:doi:10.3390/ijms19113592

31. A Nazari, Khorramdelazad H Hassanshahi G. Biological/pathological functions of the CXCL12/CXCR4/CXCR7 axes in the pathogenesis of bladder cancer. Int J Clin Oncol. (2017) 22:9911000. doi:10.1007/s10147-017-1187-x

32. D Cancilla, Rettig M P DiPersio J F. Targeting CXCR4 in AML and ALL. Front Oncol. (2020) 10:1672. doi:10.3389/fonc. 2020.01672

33. C Lu, Zhu J, Chen X, Hu Y, Xie W, Yao J,et al. Risk Stratification in Acute Myeloid Leukemia Using CXCR Gene Signatures: A Bioinformatics Analysis. Frontiers in Oncology. (2020) 10:doi:10.3389/fonc.2020.584766

34. Y Li, Reader J C, Ma X, Kundu N, Kochel T Fulton A M. Divergent roles of CXCR3 isoforms in promoting cancer stem-like cell survival and metastasis. Breast Cancer Res Treat. (2015) 149:403-15. doi:10.1007/s10549-014-3229-7

35. S Shahabuddin, Ji R, Wang P, Brailoiu E, Dun N, Yang Y,et al. CXCR3 chemokine receptor-induced chemotaxis in human airway epithelial cells: role of p38 MAPK and PI3K signaling pathways. Am J Physiol Cell Physiol. (2006) 291:C34-9. doi:10.1152/ajpcell.00441.2005

36. M G Kazanietz, Durando M Cooke M. CXCL13 and Its Receptor CXCR5 in Cancer: Inflammation, Immune Response, and Beyond. Front Endocrinol (Lausanne). (2019) 10:471. 
doi:10.3389/fendo.2019.00471

37. R Singh, Gupta P, Kloecker G H, Singh S Lillard J W, Jr. Expression and clinical significance of CXCR5/CXCL13 in human non-small cell lung carcinoma. Int J Oncol. (2014) 45:2232-40. doi:10.3892/ijo.2014.2688

38. Q Y Ma, Huang D Y, Zhang H J, Chen J, Miller W Chen X F. Function of follicular helper T cell is impaired and correlates with survival time in non-small cell lung cancer. Int Immunopharmacol. (2016) 41:1-7. doi:10.1016/j.intimp.2016.10.014

39. Q Gao, Zhao Y J, Wang X Y, Qiu S J, Shi Y H, Sun J,et al. CXCR6 upregulation contributes to a proinflammatory tumor microenvironment that drives metastasis and poor patient outcomes in hepatocellular carcinoma. Cancer Res. (2012) 72:3546-56. doi:10.1158/0008-5472.Can-11-4032

40. Y Xun, Yang H, Li J, Wu F Liu F. CXC Chemokine Receptors in the Tumor Microenvironment and an Update of Antagonist Development. Rev Physiol Biochem Pharmacol. (2020) 178:1-40. doi:10.1007/112_2020_35

\section{Tables}

Table1. Transcriptional levels of CXCR family members between normal tissues and HNSCC (ONCOMINE).

\begin{tabular}{|lllll|}
\hline Gene name & Fold change & p-value & t-test & References \\
\hline CXCR4 & 3.447 & $2.75 \mathrm{E}-13$ & 10.536 & 25 \\
\hline CXCR4 & 2.150 & $4.68 \mathrm{E}-6$ & 5.002 & 26 \\
\hline CXCR7 & 2.185 & $3.24 \mathrm{E}-7$ & 5.892 & 25 \\
\hline CXCR7 & 6.647 & 0.002 & 4.459 & 27 \\
\hline CXCR7 & 3.175 & $7.57 \mathrm{E}-4$ & 3.497 & 28 \\
\hline CXCR7 & 1.550 & $1.28 \mathrm{E}-10$ & 7.522 & 26 \\
\hline CXCR7 & 2.378 & $1.49 \mathrm{E}-4$ & 3.937 & 29 \\
\hline
\end{tabular}

Table 2. Univariate Cox proportional hazards regression analyses of $C X C R$ members and clinical features in HNSCC. 


\begin{tabular}{|llll|}
\hline \multirow{2}{*}{ Parameter } & \multicolumn{3}{l}{ Univariate analysis } \\
\cline { 2 - 4 } & $\mathrm{HR}$ & $\mathbf{9 5 \%} \mathrm{Cl}$ & $\mathrm{p}$-value \\
\hline Age & 1.024 & $1.010-1.038$ & $\mathbf{0 . 0 0 1}$ \\
\hline Gender & 0.776 & $0.566-1.063$ & 0.114 \\
\hline Grade & 1.152 & $0.917-1.449$ & 0.225 \\
\hline Stage & 1.448 & $1.203-1.743$ & $\mathbf{9 . 1 5 E}-05$ \\
\hline CXCR1 & 1.208 & $0.883-1.652$ & 0.238 \\
\hline CXCR2 & 0.809 & $0.546-1.199$ & 0.290 \\
\hline CXCR3 & 0.649 & $0.481-0.877$ & $\mathbf{0 . 0 0 5}$ \\
\hline CXCR4 & 0.529 & $0.301-0.931$ & $\mathbf{0 . 0 2 7}$ \\
\hline CXCR5 & 0.623 & $0.462-0.839$ & $\mathbf{0 . 0 0 2}$ \\
\hline CXCR6 & 0.650 & $0.474-0.892$ & $\mathbf{0 . 0 0 8}$ \\
\hline CXCR7 & 1.208 & $0.921-2.216$ & 0.111 \\
\hline
\end{tabular}

Bold means $p$-value $<0.05$.

\section{Figures}


A

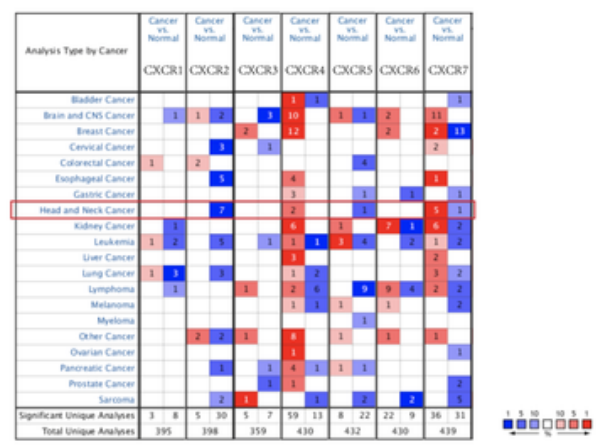

B
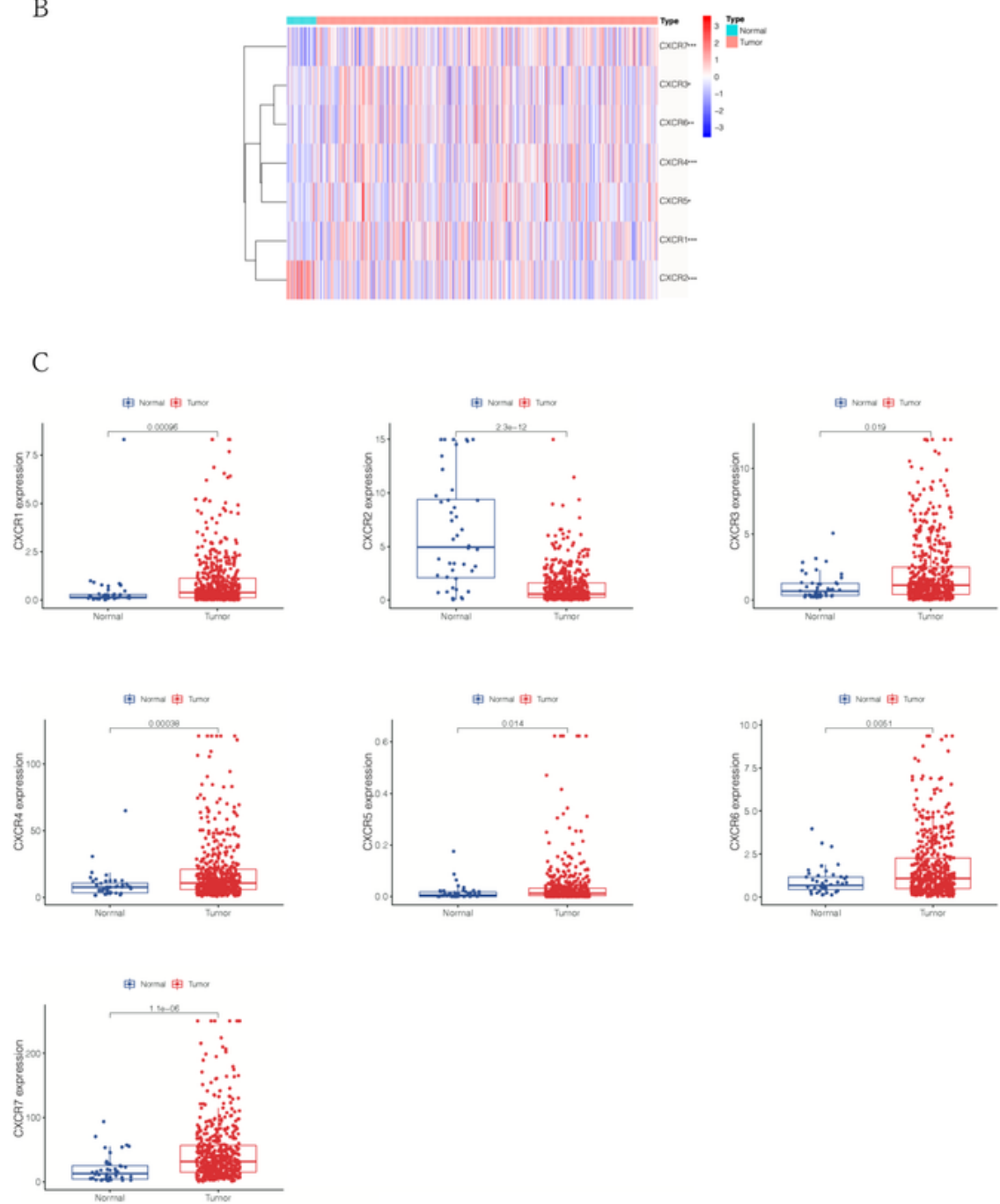

\section{Figure 1}

Expression of CXCRs in different types of cancers. (A) Red and blue in the heatmap indicate the numbers of datasets with increased and decreased levels of CXCR family members, respectively $(P<0.05)$. (B) Differential expression of CXCRs in HNSCC samples and normal tissues represented by a heatmap. The tree diagram on the left side of heatmap showed the cluster analysis between CXCR family members. *, 
$P<0.05 ; \star \star, P<0.01 ; * \star \star, P<0.001$. (C) Differential expression of CXCRs in HNSCC samples and normal tissues represented by box plots.
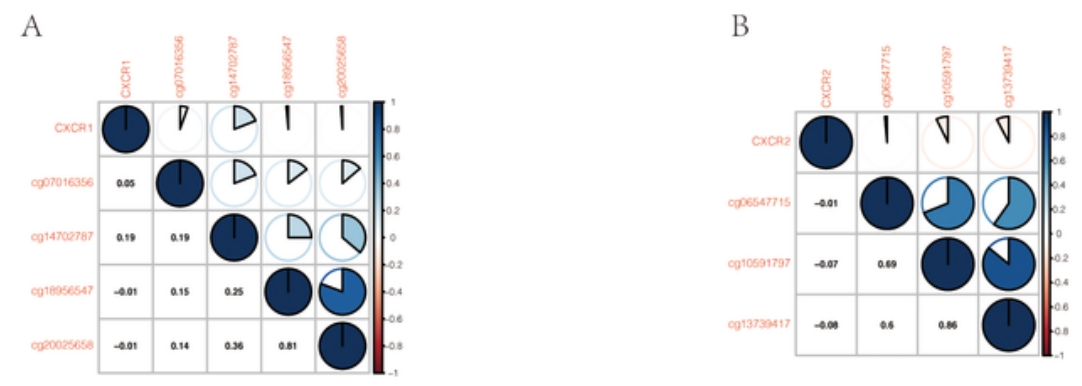

C
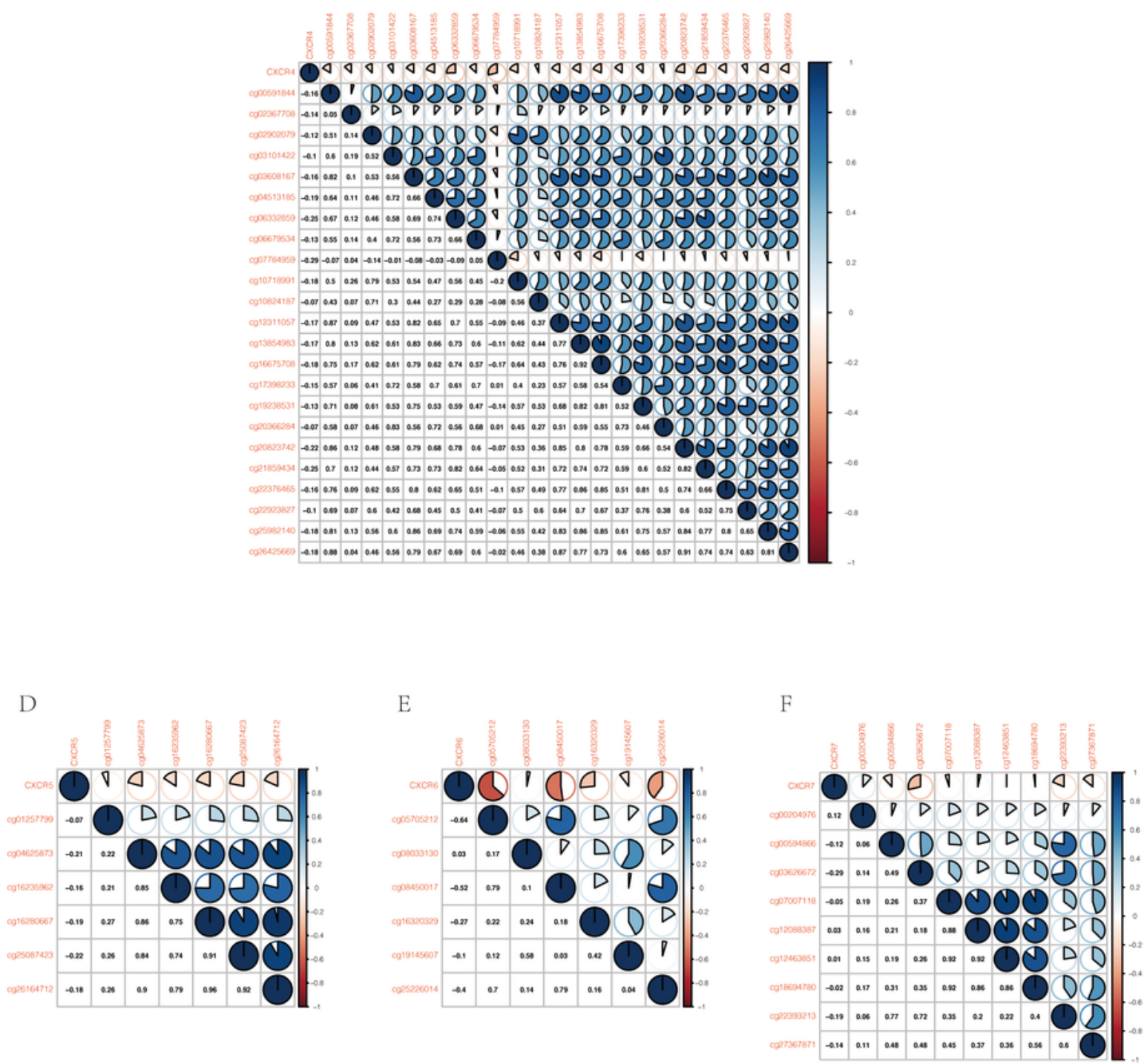

Figure 2

Correlation between methylation levels and expression of CXCRs in HNSCC. 

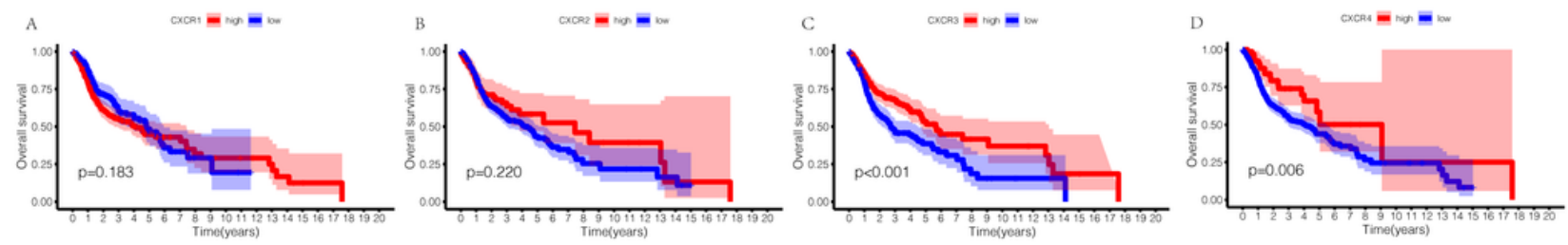

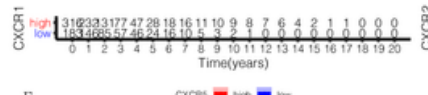
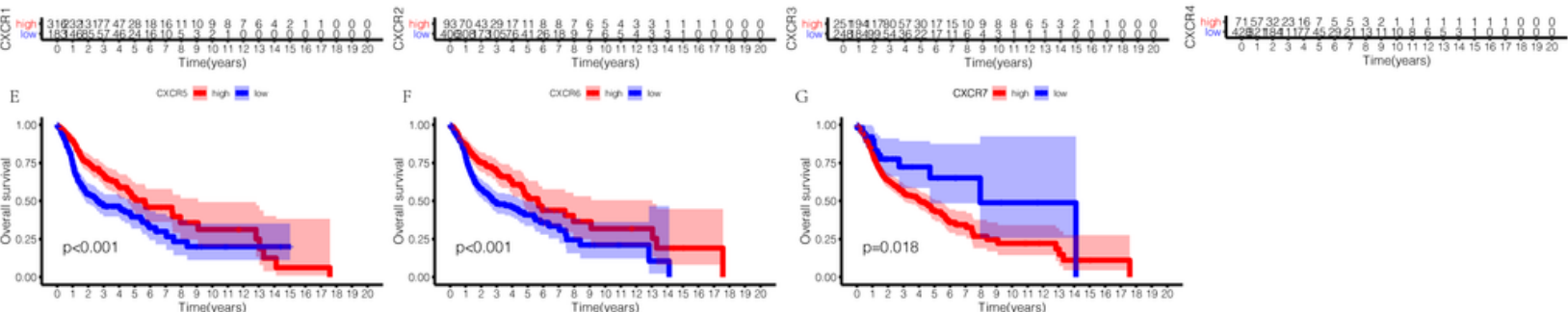

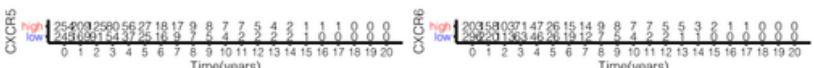
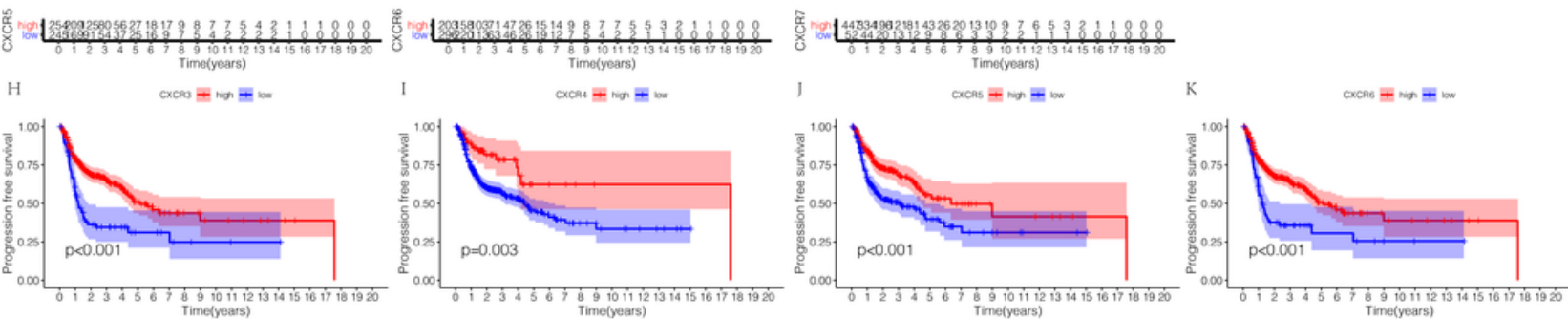

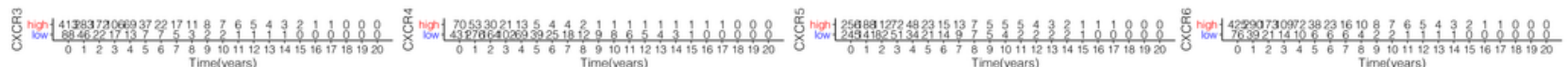

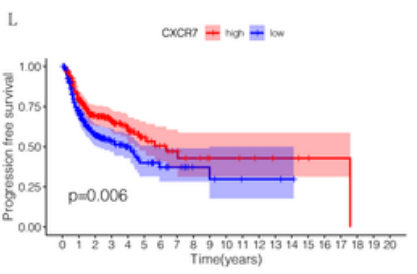

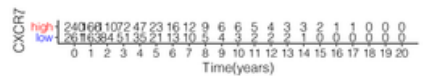

Figure 3

Prognostic value of CXCRs in HNSCC. (A-G) Survival outcomes and (H-L) Recurrence outcomes. 
A

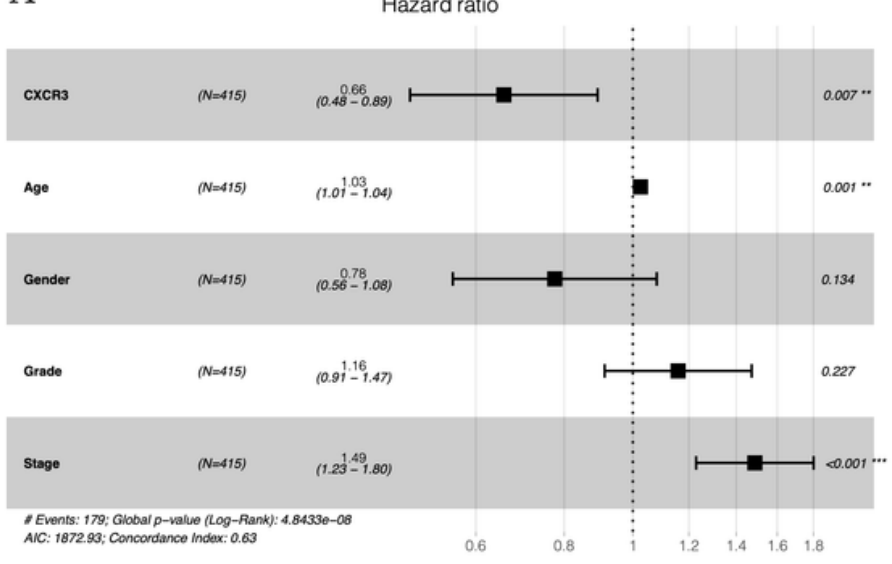

C

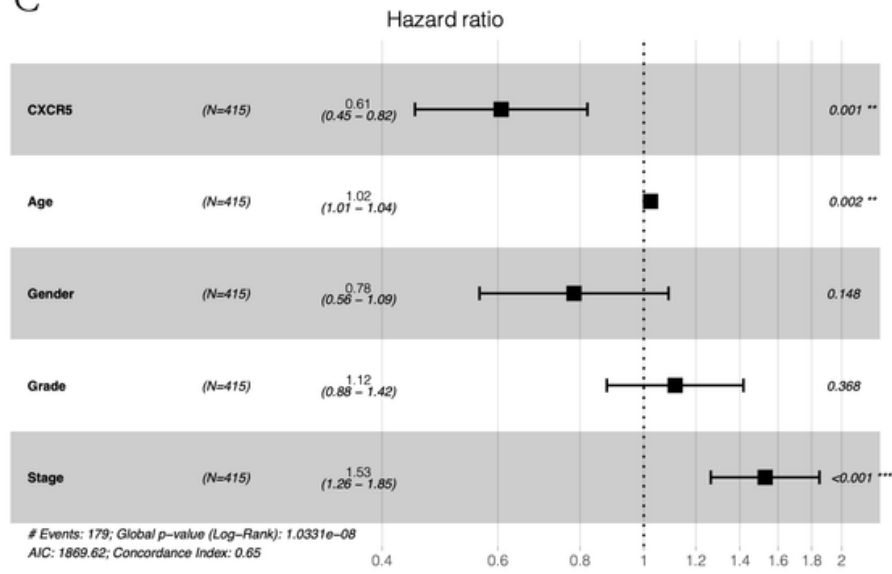

B

Hazard ratio

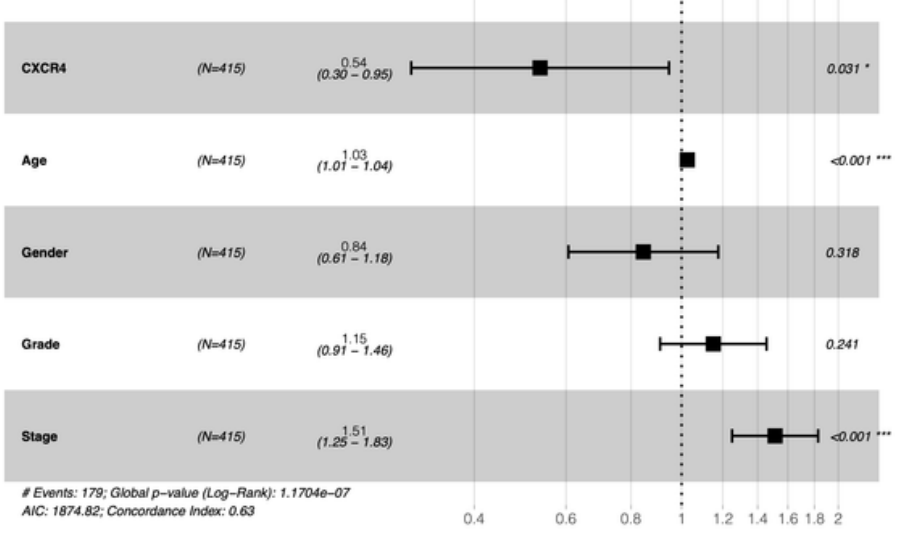

$\mathrm{D}$

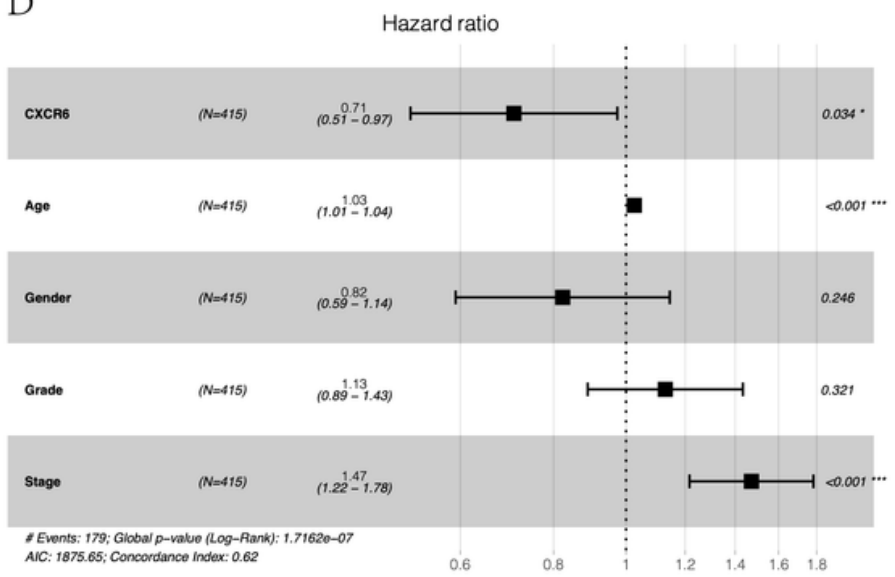

\section{Figure 4}

Results of multivariate Cox regression analyses of significant prognostic factors represented by forest plots. ${ }^{*}, \mathrm{P}<0.05$. ${ }^{*}, \mathrm{P}<0.01$. $* \star *, \mathrm{P}<0.001$. 
A

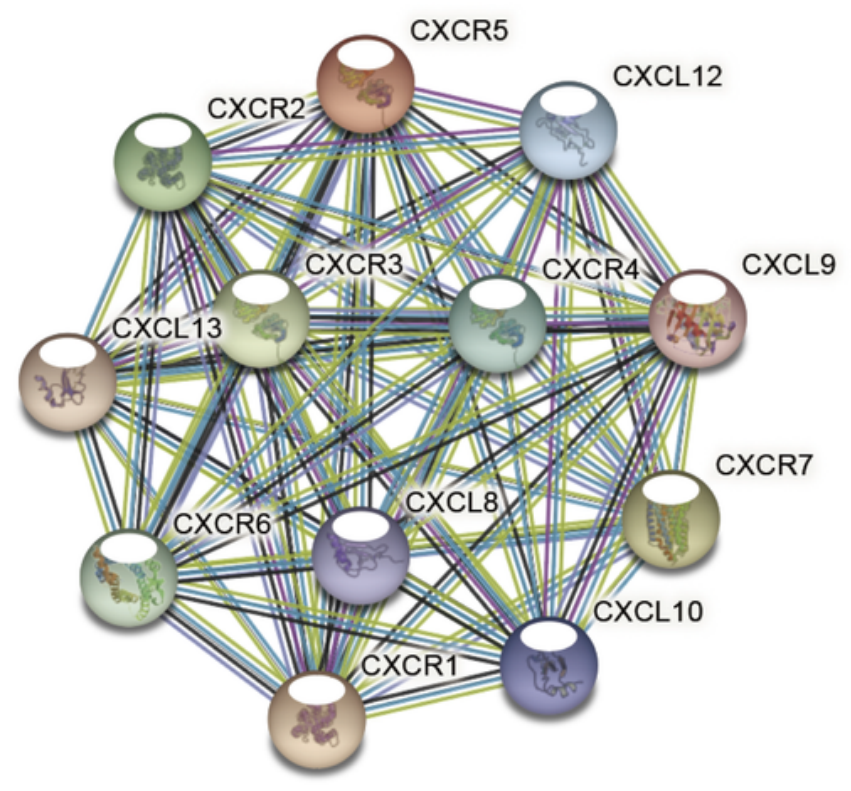

B

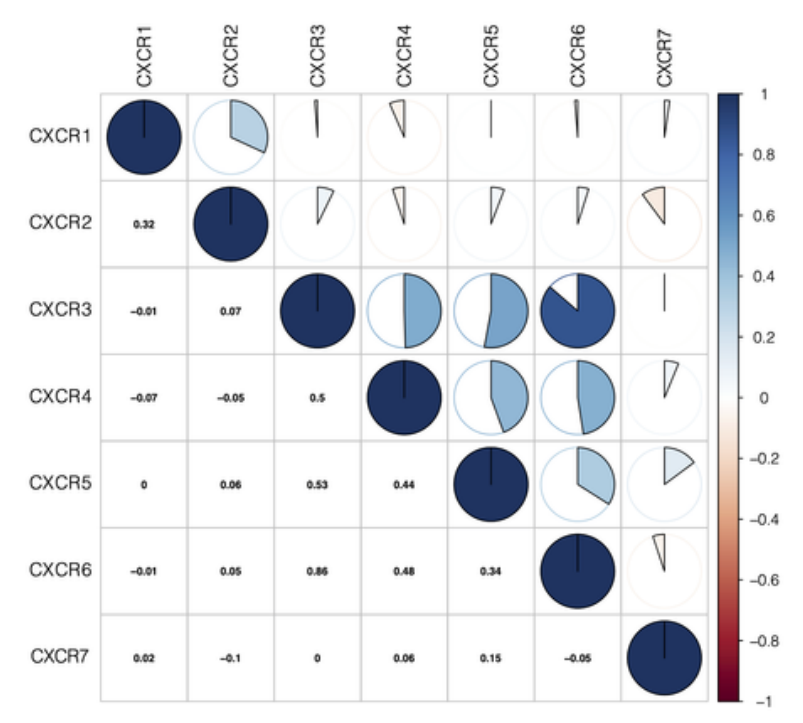

Figure 5

(A) PPI network among CXCRs. (B) Correlations between CXCRs. 
A
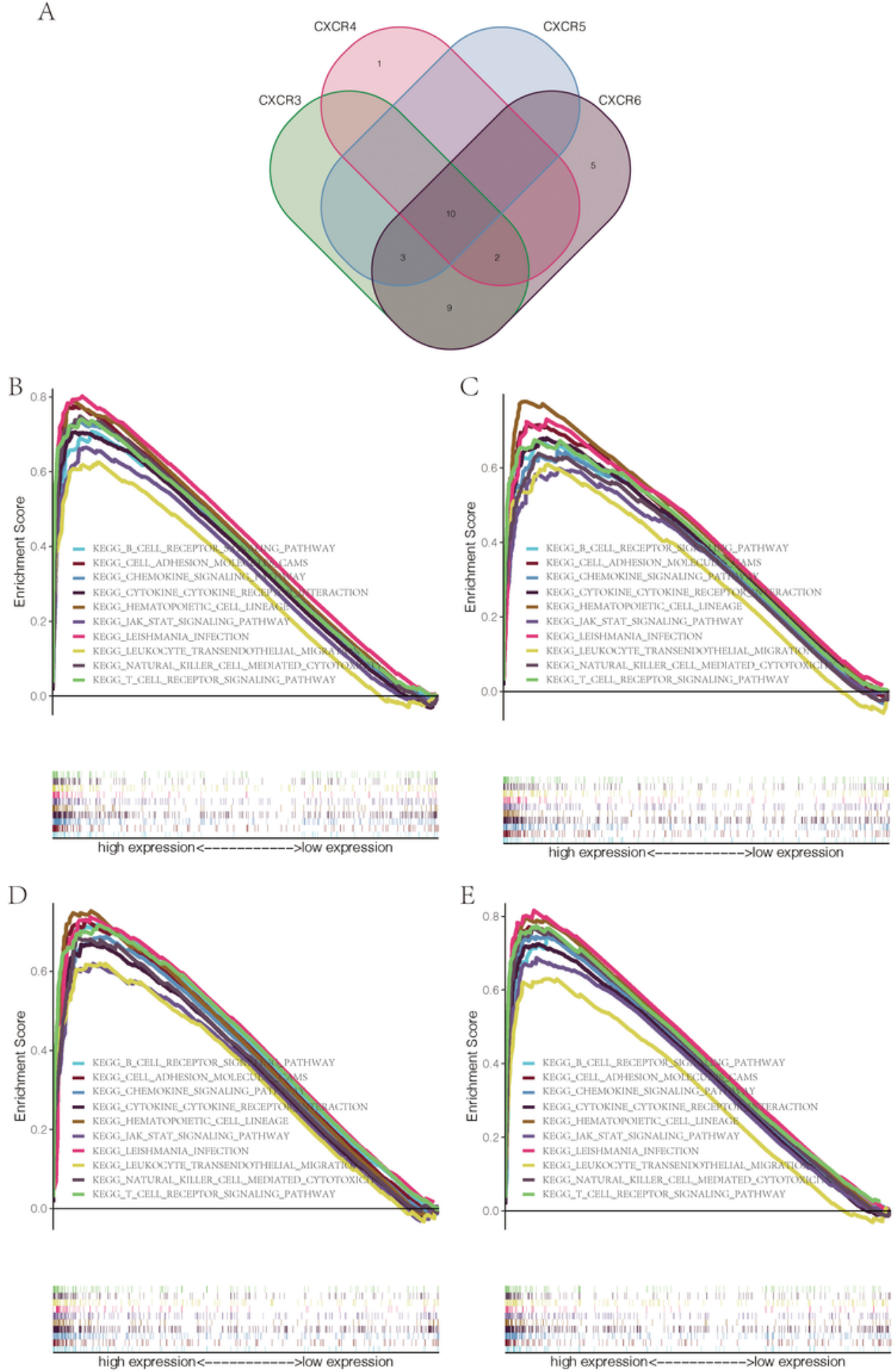

Figure 6

(A) Veen diagram showed CXCR3-6 related to 10 common enriched pathways (KEGG). Cancer-related Kyoto Encyclopedia of Genes and Genomes (KEGG) pathways associated with (B) CXCR3, (C) CXCR4, (D) CXCR5 and (E) CXCR6 based on a GSEA. 

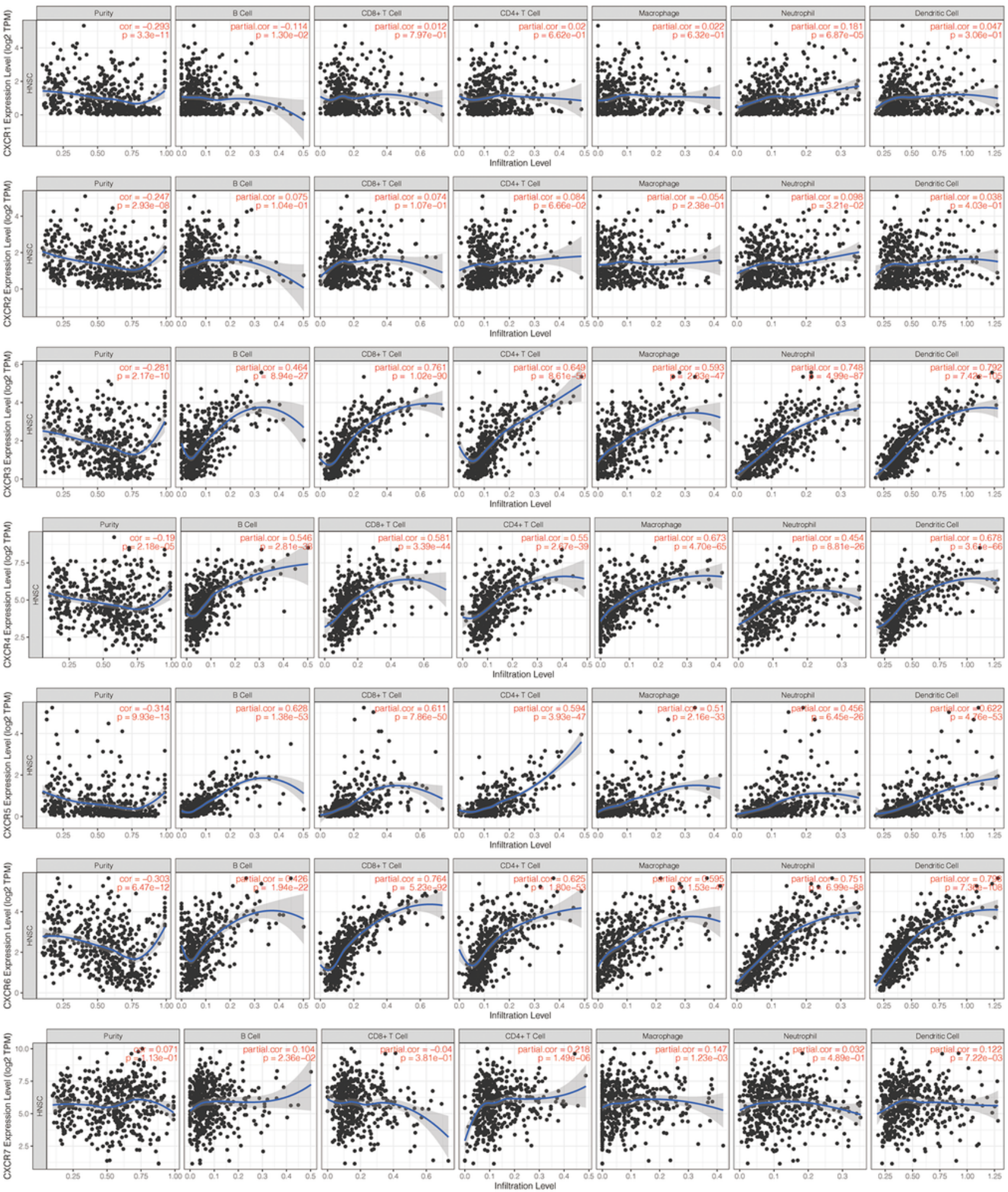

Figure 7

Correlations between tumor-infiltrating immune cells and independently prognostic CXCRs (CXCR3, CXCR4, CXCR5, and CXCR6). 

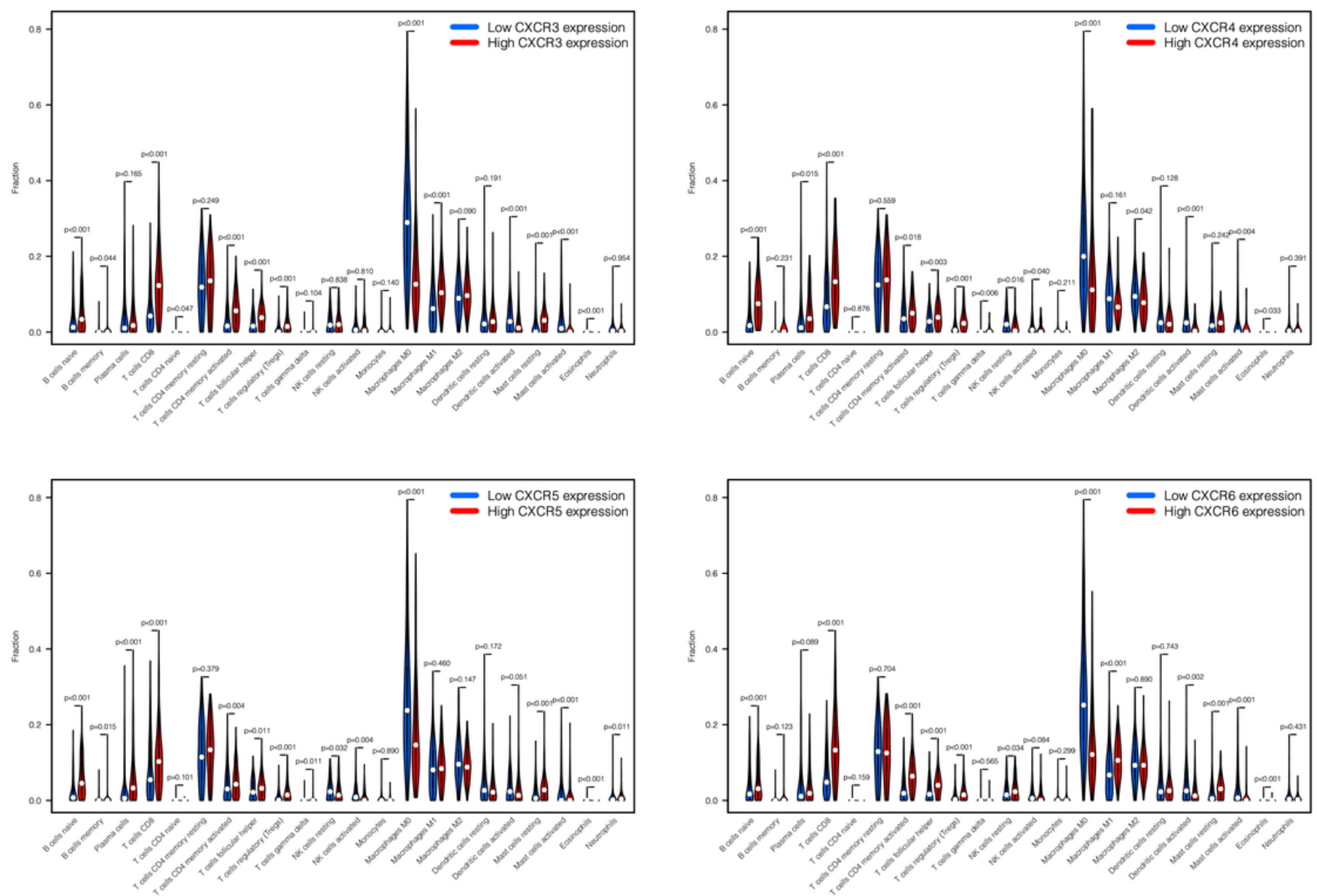

Figure 8

The abundance of tumor-infiltrating immune cells between high expression group and low expression group of CXCR3, CXCR4, CXCR5, and CXCR6 in HNSCC. 